\title{
Segmental femoral neck fracture in an elderly patient: A rare case report
}

\author{
Siddharth Kothari ${ }^{1 *}$, Atul Prakash ${ }^{2}$ \\ ${ }^{1}$ Resident, ${ }^{2}$ HOD, TATA Main Hospital, Jamshedpur, Jharkhand, India \\ *Corresponding Author: Siddharth Kothari \\ Email: siddharthkothari11@gmail.com
}

\begin{abstract}
Segmental femoral neck fracture (a combination of subcapital and intertrochanteric femoral fracture) is rare. ${ }^{1-16}$ We present a rare case of a 72 year old morbidly obese patient with an ipsilateral displaced fracture of intracapsular and Intertrochanteric femoral neck fracture.
\end{abstract}

Keywords: Segmental Femoral neck fracture, Neck and trochanter fracture.

\section{Introduction}

Intertrochanteric fracture and neck of femur fracture seperately are major causes of morbidity and mortality in elderly population throughout the world. There is bimodal distribution of these fractures, ${ }^{17,18}$ with the younger population sustaining such injuries due to high energy trauma, while in the elderly such fractures occur due to trivial trauma in the osteoporotic bones.

The combination of the above two fractures is rare. Only 16 cases have been reported in the English literature.

\section{Case History}

A 72 year old elderly, morbidly obese female with a body weight of $126 \mathrm{~kg}$ (BMI of 51.8) presented to the casualty department with a history of fall at home one week ago. The fall was from a standing height. Immediately after the fall she was unable to bear weight on the affected side.

On presentation, she was conscious and oriented. Her heart rate was 80 beats/min, and blood pressure was 150/85 $\mathrm{mm} \mathrm{Hg}$. Past medical history was significant for hypertension controlled with drugs.

On examination, the left thigh was swollen, with the leg externally rotated and shortened. There was cellulitis distal to the left calf. Range of movements of the left hip joint were painful and restricted. Venous ulcers were present on right lower limb.

$X$ rays of the pelvis with both hips and lateral $x$ ray of the left hip was done. Fig.1

A definite intertrochanteric fracture line could be seen. There was also a suscpicion of an intracapsular neck femur fracture, and an area of abnormal, well defined calcification was seen just medial to the lesser trochanter good lateral views could not be obtained because of extreme obesity. In order to get more information about the fracture and the associated heterotopic ossification, a NCCT was done.

It was a segmental neck of femur fracture was clearly evident on the NCCT. Heterotopic ossification in the tendon of the iliopsoas was seen. Fig. 2, Fig. 3

Before the injury patient was community ambulatory, walking with walker support, and had no history of any previous fracture. Given her pre-injury ambulatory status, late presentation to our centre, and her age, cemented Hemiarthroplasty with a bipolar prosthesis was chosen, rather than osteosynthesis. This would be conducive to early post op full weight bearing.

Hemiarthroplasty for neck femur fractures in the elderly has been a standard of care. Her morbid obesity made implant failure a real risk, and presence of cellulitis and venous ulcers did not augur well for early post operative weight bearing with internal fixation.

An informed consent was taken for high risk surgery.

In the operation theatre, a special table was arranged which could support her weight. The surgery was performed using the modified Hardinge approach. The middle segment comprising of the greater trochanter was clamped to the shaft, and the femoral head was excised. The femoral canal was prepared to accept the prosthesis. Care was taken to avoid cement intrusion into the fracture site. Following the insertion of the implant, head was reduced, found to be stable. We did not attempt to excise the heterotopic ossification, as it did not seem to affect the hip stability or the range of movement. Fig. 4

The Greater Trochanter was wired into place. Closure was done in layers with a drain in situ. Leg length was found to be satisfactory.

The entire procedure took $2 \mathrm{hrs}$, with an estimated blood loss of $300 \mathrm{ml}$.

Patient was shifted to Intensive care post operatively, and was intubated with NIV support as planned. She maintained vitals and was stable in the immediate post op period. Post op Hb was $7.7 \mathrm{~g} / \mathrm{dl}$. Two units of packed cells were transfused. Other reports and parameters were stable.

She was extubated after $36 \mathrm{hrs}$. She was shifted to the orthopaedic ward on the 3rd day. In bed mobilisation was started on the $3^{\text {rd }}$ day. Post operative $\mathrm{x}$-ray was done and found to be satisfactory with good restoration of the Shenton's arc. Fig. 5

She was moblised in the wards. Knee and ankle range of movement exercises were also instituted.

Sutures were removed on the $14^{\text {th }}$ post op day. The surgical site was dry throughout. Patient was discharged on the $15^{\text {th }}$ day, walking with walker support.

She followed up regularly at 6 weeks and 3 months interval. At her $3^{\text {rd }}$ month follow up, the $x$ ray showed wire breakage along with loss of capture of the GT. She continued to walk with the help of a walker without any complaints and showed no clinical signs of instability. 


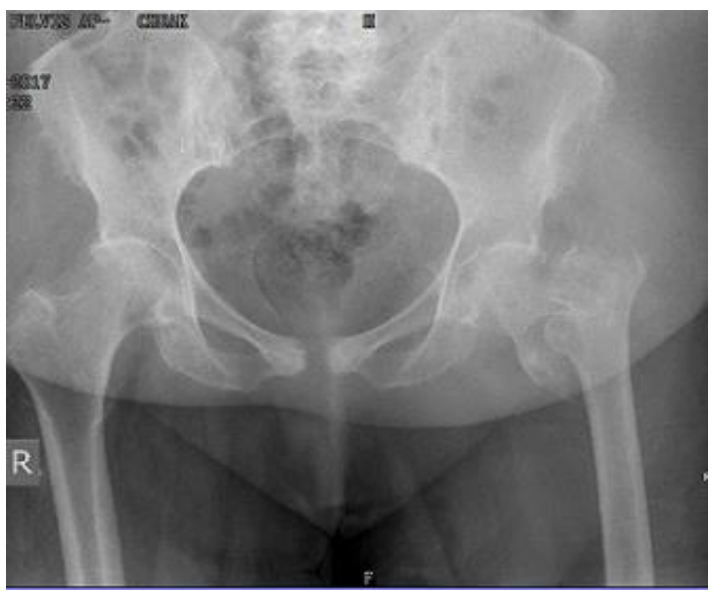

Fig. 1: Pre operative $x$ ray showing fracture line along intertrochanteric region and neck of femur with calcification present medial to the lesser trochanter

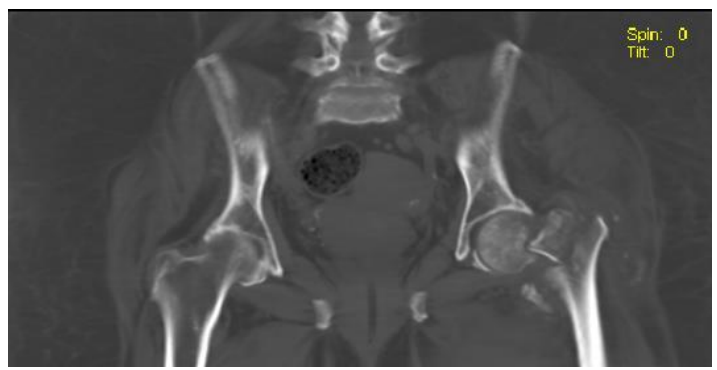

Fig. 2: CT cut sections showing clearly both fracture lines

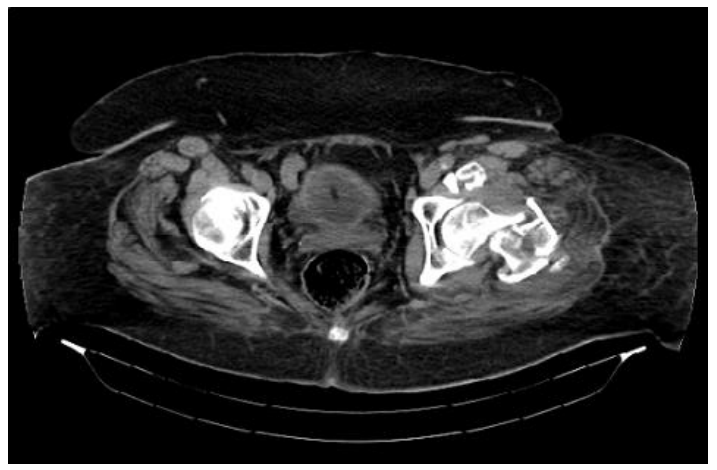

Fig. 3: CT cut sections showing clearly both fracture lines

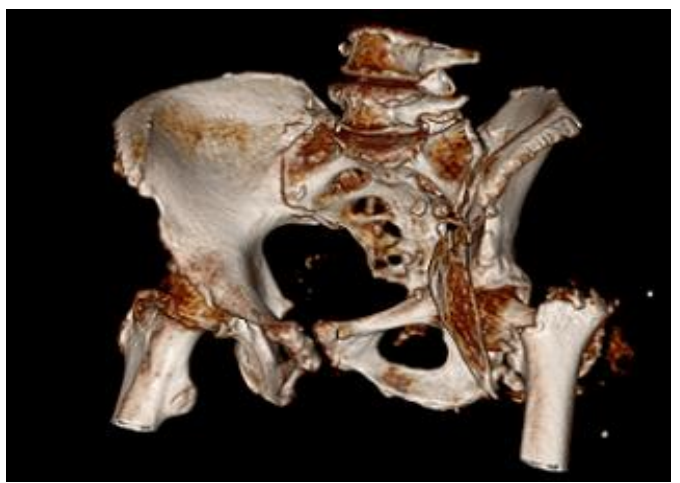

Fig. 4: Three-D reconstruction image showing the area of heterotopic ossification medial to the lesser trochanter

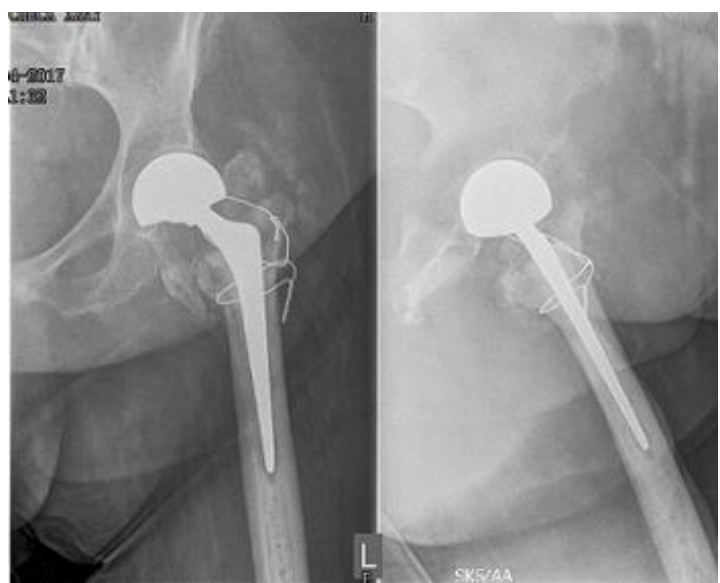

Fig. 5: Post operative $x$ ray after hemiarthroplasty and Greater Trochanteric wiring

\section{Discussion}

Segmental femur neck fractures are a rare entity. Till date, there have been 16 reports published in the literature. ${ }^{1-16}$

These fractures show a bimodal distribution. Out of the sixteen, five patients were in the younger age group, and presented with history of high energy trauma.

Only three cases amongst the sixteen, utilised a CT to confirm their diagnosis. It was also on CT that the complete fracture profile could be recognised in this case. We advise a CT in all doubtful cases

All patient in the young age group were managed with internal fixation/osteosynthesis. Nine cases were seen in the elderly population. These all involved low energy mechanisms. Out of all the elderly patients, eight were fixed with internal fixation. Three were managed with hemiarthroplasty.

The case presented by us is of an elderly, morbidly obese female patient with osteoporosis. It was associated with a low energy fall. There was delayed presentation. She was morbidly obese with a BMI of 51.85. Her case would be $17^{\text {th }}$ in the series, and $4^{\text {th }}$ such case managed with hemiarthroplasty

Our choice of implant was based on the fracture pattern, delayed presentation, age of the patient and need for early full weight bearing.

Patient had good functional outcome, and was walking well. Hemiarthroplasty is a good implant choice for elderly patient with this fracture profile.

\section{Conclusion}

Ipsilateral Intertrochanteric and neck femur fractures are rare. Their diagnosis require keen observation of the $\mathrm{x}$ ray and concomitant CT imaging. The choice of implant/prosthesis has to be individualised.

\section{Patient Declaration Statement}

"The authors certify that they have obtained all appropriate patient consent forms. In the form the patient(s) has/have given his/her/their consent for his/her/their images and other 
clinical information to be reported in the journal. The patients understand that their names and initials will not be published and due efforts will be made to conceal their identity, but anonymity cannot be guaranteed."

\section{References}

1. Pemberton D.J., Kriebich D.N., Moran C.G. Segmental fracture of the neck of the femur. Injury 1989

2. An H.S., Wojcieszek J.M., Cooke R.F., Limbird R., Jackson W.T. Simultaneous ipsilateral intertrochanteric and subcapital fracture of the hip. A case report. Orthop 1989.

3. Lawrence B., Isaacs C. Concomitant ipsilateral intertrochanteric and subcapital fracture of the hip. J Orthop Trauma 1993.

4. Cohen I., Rzetelny V. Simultaneous ipsilateral pertrochanteric and subcapital fractures. Orthop 1999.

5. Yuzo O., Yamanaka M., Hiroshi T., Naoyoshi I. A case of femoral neck and trochanteric fracture in ipsilateral femur. Orthop Traumatol 2001

6. Kumar R., Khan R., Moholkar K., Smyth H., Borton D. A rare combination fracture of the neck of femur. Eur J Orthop Surg Traumatol 2001

7. Lakshmanan P., Peehal J.P. Management of an unusual intraand extra-capsular subcapital femoral neck fracture. Acta Orthop Belg 2005.

8. Sayegh F., Karataglis D., Trapotsis S., Christopforides J., Pournaras J. Concomitant ipsilateral pertrochanteric and subcapital fracture of the proximal femur. Eur J Trauma 2005.

9. Poulter R.J., Ashworth M.J. Concomitant ipsilateral subcapital and intertrochanteric fractures of the femur. Inj Extra 2007.

10. Butt M.F., Dhar S.A., Hussain A., Gani N.U., Kangoo K.A., Farooq M. Femoral neck fracture with ipsilateral trochanteric fracture: is there room for osteosynthesis? Internet J Orthop Surg 2007.
11. Perry D.C., Scott S.J. Concomitant ipsilateral intracapsular and extracapsular femoral neck fracture: a case report. J Med Case Rep 2008.

12. Dhar S.A., Mir M.R., Butt M.F., Farooq M., Ali M.F. Osteosynthesis for a T-shaped fracture of the femoral neck and trochanter: a case report. J Orthop Surg 2008.

13. Loupasis G., Ntagiopoulos P.G., Asimakopoulos A. Concomitant ipsilateral subcapital and intertrochanteric fractures of the femur: a case report. J Med Case Rep 2010.

14. Neogi D.S., Ajay Kumar K.V., Trikha V., Yadav C.S. Ipsilateral femoral neck and trochanter fracture. Indian $J$ Orthop 2011.

15. Muzaffar N., Bashir N., Ahmad A., Ahmad N., Hafeez A. Lpsilateral fracture of the femoral neck, trochanter and shaft: a unique case. Webmed Central Orthop 2011.

16. Tahir M, Lakkol S, Naique S.

17. Segmental neck of femur fractures: A unique case report of an ipsilateral subcapital, greater trochanteric and intertrochanteric fracture and proposed management algorithm. Int J Surg Case Rep 2014.

18. Roberts SE, Goldacre MJ. Time trends and demography of mortality after fractured neck of femur in an English population, 1968-98: database study. BMJ 2003;327(7418):771-5.

19. Zuckerman JD. Hip fracture. N Engl J Med 1996.

How to cite this article: Kothari $\mathrm{S}$, Prakash $\mathrm{A}$. Segmental femoral neck fracture in an elderly patient: A rare case report. Indian J Orthop Surg 2019;5(2):162-4. 